\title{
Bias-Driven Conductance Increase with Length in Porphyrin Tapes
}

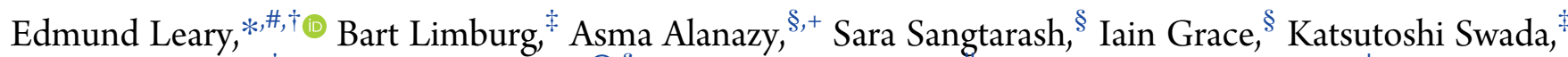
Louisa J. Esdaile, ${ }^{\ddagger}$ Mohammed Noori, ${ }^{\otimes}$, M. Teresa González, ${ }^{\Uparrow}$ Gabino Rubio-Bollinger, ${ }^{\perp}$

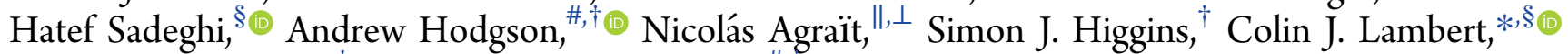
Harry L. Anderson, $*, \neq \odot$ and Richard J. Nichols $*, \#, \uparrow$

${ }^{\dagger}$ Department of Chemistry, Donnan and Robert Robinson Laboratories, University of Liverpool, Liverpool L69 7ZD, United Kingdom

${ }^{\#}$ Surface Science Research Centre and Department of Chemistry, University of Liverpool, Oxford Street, Liverpool L69 3BX, United Kingdom

${ }^{\ddagger}$ Department of Chemistry, Chemistry Research Laboratory, Oxford University, Oxford OX1 3TA, United Kingdom

${ }^{\S}$ Department of Physics, Lancaster University, Lancaster LA1 4YW, United Kingdom

${ }^{\otimes}$ Physics Department, College of Science, University of Thi Qar, Thi Qar 0964, Iraq

${ }^{+}$Department of Mathematics and Statistics, Lancaster University, Lancaster LA1 4YW, United Kingdom

"Instituto Madrileño de Estudios Avanzados (IMDEA), Calle Faraday 9, Campus Universitario de Cantoblanco, 28049 Madrid, Spain

${ }^{\perp}$ Departamento de Física de la Materia Condensada, IFIMAC and Instituto "Nicolás Cabrera”, Universidad Autónoma de Madrid, E-28049 Madrid, Spain

Supporting Information

ABSTRACT: A key goal in molecular electronics has been to find molecules that facilitate efficient charge transport over long distances. Normally, molecular wires become less conductive with increasing length. Here, we report a series of fused porphyrin oligomers for which the conductance increases substantially with length by $>10$-fold at a bias of $0.7 \mathrm{~V}$. This exceptional behavior can be attributed to the rapid decrease of the HOMO-LUMO gap with the length of fused porphyrins. In contrast, for butadiyne-linked porphyrin oligomers with moderate inter-ring coupling, a normal conductance decrease with length is found for all bias voltages explored $( \pm 1 \mathrm{~V})$, although the attenuation factor $(\beta)$ decreases from ca. $2 \mathrm{~nm}^{-1}$ at low bias to $<1 \mathrm{~nm}^{-1}$ at $0.9 \mathrm{~V}$, highlighting that $\beta$ is not an intrinsic molecular property. Further theoretical analysis using density functional theory underlines the role of intersite coupling and indicates that this large increase in conductance with length

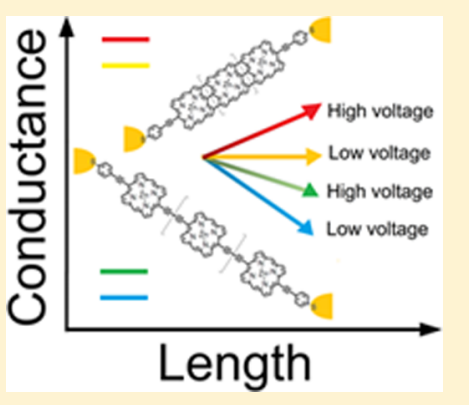
at increasing voltages can be generalized to other molecular oligomers.

\section{INTRODUCTION}

Investigating length dependence and long-range charge transport across individual molecules is an important area of study related to many chemical and physical processes. One example is in photosynthesis, where the harvesting of sunlight is achieved via stepwise electron transfer. ${ }^{1}$ Another is the study of electron transport through protein-based junctions, which is found to be surprisingly efficient, and where the exact transport mechanism remains unclear. ${ }^{2,3}$ Single molecule-based devices offer benefits such as switchability, ${ }^{4-8}$ reduced power requirements and small footprints, and have the potential to transform areas such as chemical sensing, molecular logic and thermoelectric devices. ${ }^{9-11}$ Porphyrins, which are an important part of the photosynthetic process, ${ }^{1}$ are promising candidates for sub$10 \mathrm{~nm}$ electronics due to their long-range charge transport ability. ${ }^{12-22}$ They are planar, aromatic macrocycles, and when joined together in the form of oligomers, the degree of overall conjugation, and hence the HOMO-LUMO gap, depends on the type of inter-ring connection. Connection at the meso positions with alkynes results in moderate electronic communication between rings. ${ }^{23-25}$ Linking with multiple covalent bonds, on the other hand, produces much stronger effects. ${ }^{18}$ Triply linked (edge-fused) porphyrin tapes show remarkable electronic properties, and dramatic reductions in HOMO-LUMO gap with length, with some of the smallest gaps reported for organic compounds. ${ }^{26}$ This makes them extremely interesting to study both from a fundamental point of view, to test our models of electron transfer, and more pragmatically, to test their ability as molecular wires. To the best of our knowledge, however, there have been only a couple of experimental studies into the conductance of fused porphyrins with well-defined anchor groups ${ }^{13,27}$ and only one theoretical study. ${ }^{28}$ Furthermore, there are no systematic

Received: June 15, 2018

Published: September 12, 2018 

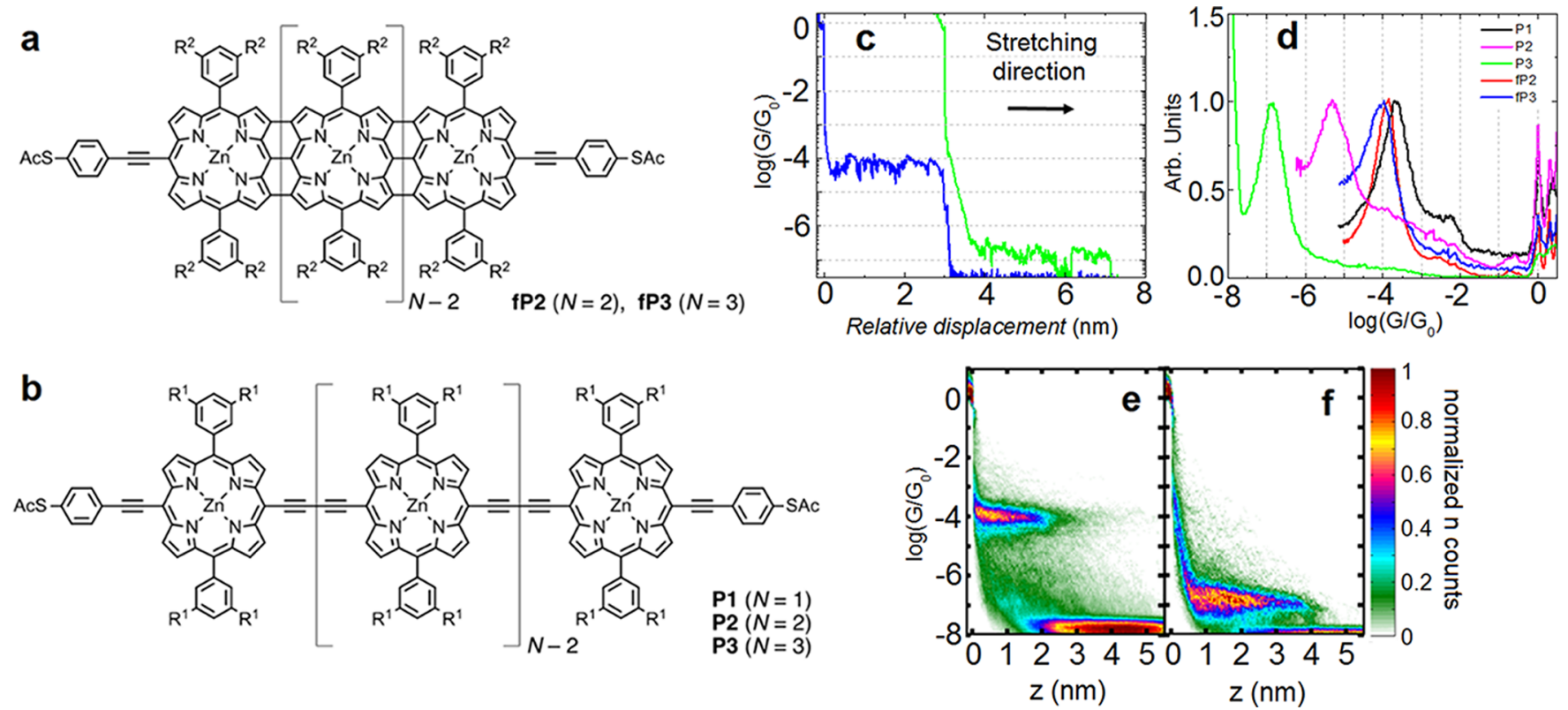

Figure 1. (a) Structure of fused porphyrins fP2 and fP3 $\left(\mathrm{R}^{2}=\mathrm{Si}\left(\mathrm{C}_{6} \mathrm{H}_{13}\right)_{3}\right)$ and (b) butadiyne-linked porphyrins $\mathbf{P 1}, \mathbf{P 2}$ and $\mathbf{P 3}\left(\mathrm{R}^{1}=\mathrm{OC}_{8} \mathrm{H}_{17}\right)$. (c) Individual $G-z$ traces for $\mathrm{PP} 3$ at $0.1 \mathrm{~V}$ (blue trace, left) and $\mathbf{P} 3$ at $0.2 \mathrm{~V}$ (green trace, right). (d) $1 \mathrm{D}$ low bias conductance histograms for each compound normalized to have a peak height of 1 . (e) $2 \mathrm{D}$ histogram of 1600 plateau-containing traces for fP3. (f) $2 \mathrm{D}$ histogram of 622 plateaucontaining traces for $\mathbf{P} 3$.

experimental or theoretical studies of the effects of applied bias voltage on the length dependence of their conductance. Systematic studies into distance dependence as a function of voltage are themselves rare, with just a few examples in the literature, mostly without well-defined anchor groups. ${ }^{29-31}$ It is therefore of great interest to study how the change in conductance with length of oligo(porphyrin)s with welldefined anchor groups depends on voltage.

In general, for coherent transport, molecular conductance is expected to decrease exponentially with length, following the form:

$$
G(l)=A \exp (-\beta l)
$$

where $l$ represents the molecular length, $A$ is a prefactor that sets the order of magnitude and $\beta$ is the conductance attenuation factor that describes the degree to which the conductance decays as the length of the wire is increased. ${ }^{21}$ For single molecules wired between a pair of metallic (normally gold) electrodes, alkanes display high $\beta$ values, between 8 and $10 \mathrm{~nm}^{-1}$, 32,33 whereas oligo(phenyl)s and oligo(phenylene ethynylene)s are much lower, between 3 and $4 \mathrm{~nm}^{-1} \cdot 34,35$ This trend clearly demonstrates that conjugation through $\pi$-bonding produces lower $\beta$ values than $\sigma$-bonding, highlighting the importance of chemical structure on conductance attenuation. As conductance, however, is expected to change with voltage, it is natural to ask how the attenuation varies as a consequence. Our recent theoretical analysis of zero-bias transport through fused porphyrin wires, ${ }^{28}$ predicts that the zero bias $\beta$ is sensitive to the anchor group, and for fused porphyrins connected to graphene electrodes the zero bias conductance can increase with increasing length. This "negative $\beta$ " is due to the quantum nature of electron transport through such wires, arising from the strong narrowing of the HOMO-LUMO gap as the length of the oligomers increases. Because the transmission coefficient depends strongly on the energy of injected electrons, a significant voltage dependence of $\beta$ is anticipated.

We have synthesized two families of porphyrin oligomers, one with moderate inter-porphyrin coupling, and the other with strong coupling. Moderate coupling is achieved via butadiyne (C4) linkers (P1-P3 as shown in Figure 1b), and much stronger coupling is achieved by directly fusing the porphyrins, creating the structures shown in Figure 1a (fP2 and fP3). The HOMO-LUMO gap decreases with length in both series, with the largest reduction seen for the fused series. The electrochemical HOMO-LUMO gaps for fP2 and fP3 were measured to be 1.08 and $0.78 \mathrm{eV}$, respectively (see Table S1 and Figure S2 in the Supporting Information for the cyclic voltammetry data). For reference, the electrochemistry of butadiyne-linked oligoporphyrins was studied previously by Winters et al. ${ }^{14}$ To bind the porphyrin units to the electrodes, we have prepared the molecules with thioacetate groups attached to phenylacetylene groups, which cleave to produce $\mathrm{Au}-\mathrm{S}$ bonds on the gold surface, ${ }^{36}$ and which we, and others, have used previously for porphyrin molecular wires. ${ }^{37,38}$

\section{RESULTS AND DISCUSSION}

Measuring the Low-Bias Single Molecule Conductance. We measured the low-bias conductance of each compound at a certain applied bias voltage $(0.2 \mathrm{~V}$ for P1-3 and $0.1 \mathrm{~V}$ for $\mathbf{f P 2} / 3$ ) using a home-built scanning tunneling microscope (STM), employing the break junction technique. We performed thousands of open-close cycles on each sample and focused on the opening stage of the measurement. For more details on the sample preparation and the complete methodology, please see the Supporting Information (SI Section 2). Example $G-z$ (conductance-distance) traces for fP3 and P3 can be seen in Figure 1c, with the corresponding 2D histograms in Figure 1e,f. The 1D histograms for each compound are shown in Figure 1d, where each peak maximum is normalized to a value of 1 to facilitate comparison of the 
most probable conductance (the original histograms normalized according to the procedure described in reference ${ }^{39}$ can be found in Figure S4 in the Supporting Information). To extract a conductance value for each compound, we fit a single Gaussian curve to each 1D histogram and extract the peak position. A summary of the low-bias conductance values is presented in Table 1, with 2D histograms also shown in Figure S5.

Table 1. Measured Low-Bias Single Molecule Conductance Values (from large datasets) and Calculated Junction Lengths $^{a}$

$\begin{array}{ccc}\text { Molecule } & \text { Low-bias conductance }\left(\log \left(G / G_{0}\right)\right) & \mathrm{Au}-\mathrm{Au} \text { distance }(\mathrm{nm}) \\ \text { P1 } & -3.7(0.8) & 2.6 \\ \text { P2 } & -5.3(0.8) & 3.9 \\ \text { P3 } & -6.9(0.7) & 5.2 \\ \text { fP2 } & -3.9(0.8) & 3.4 \\ \text { fP3 } & -4.1(0.7) & 4.2\end{array}$

${ }^{a} \mathbf{P 1} / \mathbf{P 2} / \mathbf{P} 3$ were measured at $0.2 \mathrm{~V}, \mathbf{f P 2}$ and $\mathbf{f P 3}$ were measured at $0.1 \mathrm{~V}$. The values in parentheses are the FWHM. The $\mathrm{Au}-\mathrm{Au}$ distance is the calculated separation between two gold atoms attached to the two terminal sulfur atoms.

It is clear that the conductance of the butadiyne-linked oligomers decays rapidly with length at low bias, whereas for the fused wires an almost constant conductance is found across the series. We evaluated the low-bias $\beta$ value for the butadiyne series to be $2.7( \pm 0.1) \mathrm{nm}^{-1}$, where the error is that of the linear fit. For the fused series, $\beta$ is significantly lower at 0.53 $( \pm 0.06) \mathrm{nm}^{-1}$. For further discussion about the uncertainties associated with these values, please see Section 2.4 in the Supporting Information.

Voltage Dependent Transport Behavior. After establishing the low-bias behavior, we set out to test how the conductance versus length relation depends on voltage. We conducted a series of $I-V$ measurements for each compound during the stretching of a molecular junction (several hundred junctions were tested per compound, and the experiments were carried out on the same samples as for the fixed-bias measurements). $I-V$ curves were recorded at set intervals along a trace, meaning that for each junction we collected on average between 30 and 50 curves. We waited until a minimum threshold distance was crossed (at least $0.4-0.5 \mathrm{~nm}$ ) for each junction before starting each $I-V$ measurement in order to avoid too high electric fields, which we find can cause premature junction cleavage. Further details about the measurement can be found in SI Section 2.3, including a table of measurement parameters (gains and series resistors employed; Table S2) and the plateau length histograms (Figure S12). Figure 2a shows an example of an individual G$z$ plateau for an fP3 molecule in which $I-V$ traces were recorded (starting at a length of $0.8 \mathrm{~nm}$ and finishing at the end of the plateau). Figure $2 \mathrm{~b}$ shows examples of $I-V$ traces (plotted as $\log \left(G / G_{0}\right)-V$, where $G=I / V$ ) that were recorded during the red highlighted section in panel a. Further individual examples for each compound are shown in Figures S13-17. Figure S11a-e shows the $2 \mathrm{D} G-z$ histograms for all the plateau-containing traces at the same bias voltage as used in the fixed-bias measurement $(0.2 \mathrm{~V}$ for $\mathbf{P 1}-3$ and $0.1 \mathrm{~V}$ for fP2/3).

Figure $3 \mathrm{a}-\mathrm{e}$ shows the 2D-histograms derived from all $I-V$ curves recorded during the stretching of each junction (plotted
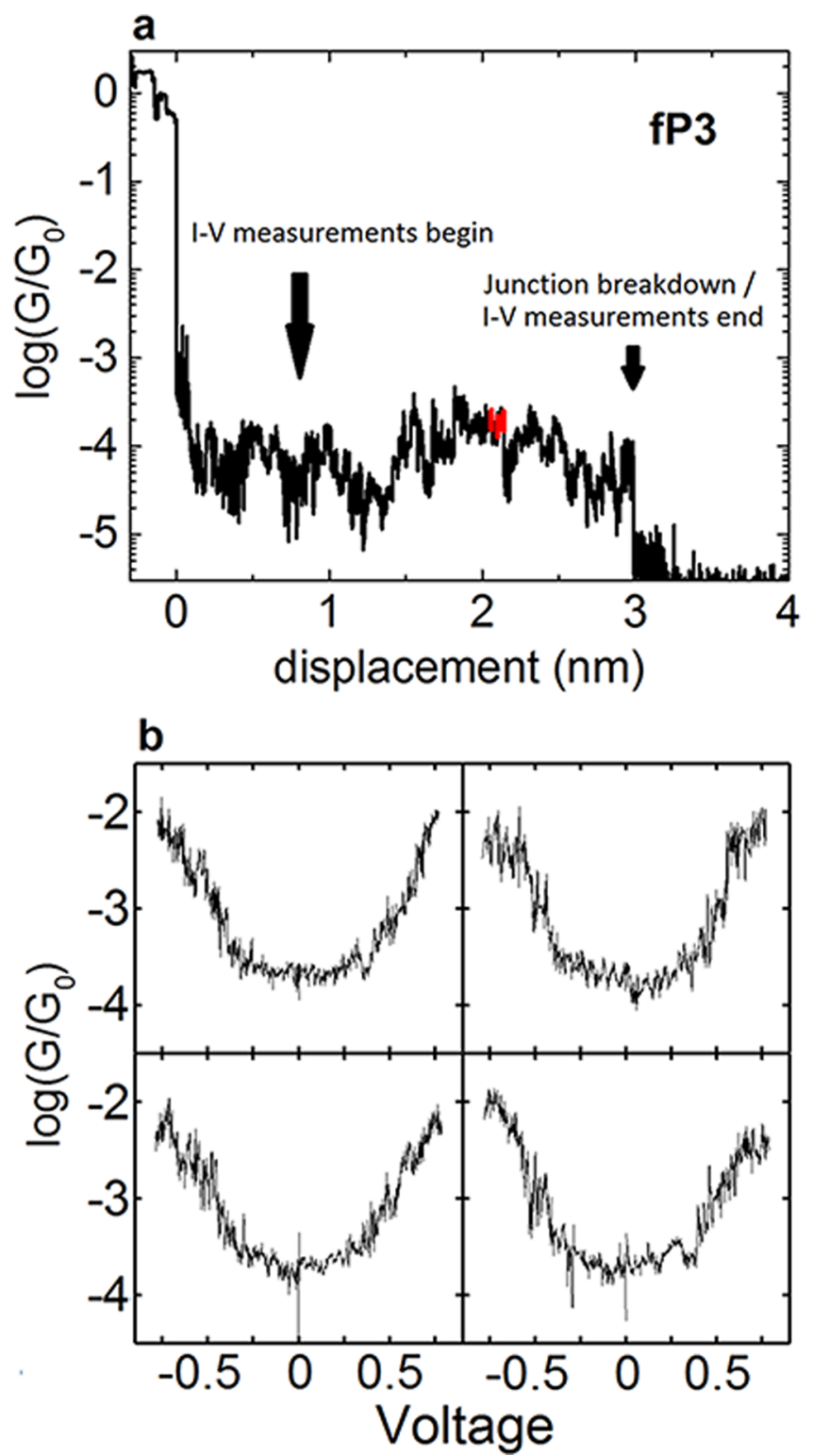

Figure 2. (a) Example $G-z$ trace for fP3 over which $I-V$ bias sweeps were performed. (b) Examples of $I-V$ curves plotted as $\log \left(G / G_{0}\right)$ taken from the red highlighted region in panel a.

as $\left.\log \left(G / G_{0}\right)-V\right)$. The conductance increases with voltage for all compounds, but the magnitude of increase becomes greater as the number of porphyrin units increases. The monomer P1 shows the smallest difference between low and high bias (an increase of $\Delta \log \left(G / G_{0}\right)=0.2$ between 0.1 and $0.7 \mathrm{~V})$. Conversely, the conductance of $\mathbf{P} 3$ rises by $\Delta \log (G /$ $\left.G_{0}\right)=1.0$ over the same voltage range. For the fused series, the $G-V$ dependence is even more pronounced, and $\mathbf{f P} 3$ increases in conductance by $\Delta \log \left(G / G_{0}\right)=1.3$ between 0.1 and $0.7 \mathrm{~V}$, a larger increase over the same range compared to P3.

Figure $4 \mathrm{a}, \mathrm{b}$ shows $1 \mathrm{D}$ histograms at selected voltages $(0.1$ and $0.7 \mathrm{~V})$. The peak positions at these voltages are plotted in Figure $4 \mathrm{c}$ as a function of the maximum theoretical junction length (measured between two $\mathrm{Au}$ atoms attached to the terminal $S$ atoms using molecular modeling). At or below 0.1 V, P1 has the highest conductance, with fP2 and fP3 both being very similar. This is consistent with the fixed-bias measurement considering the experimental uncertainty (which 


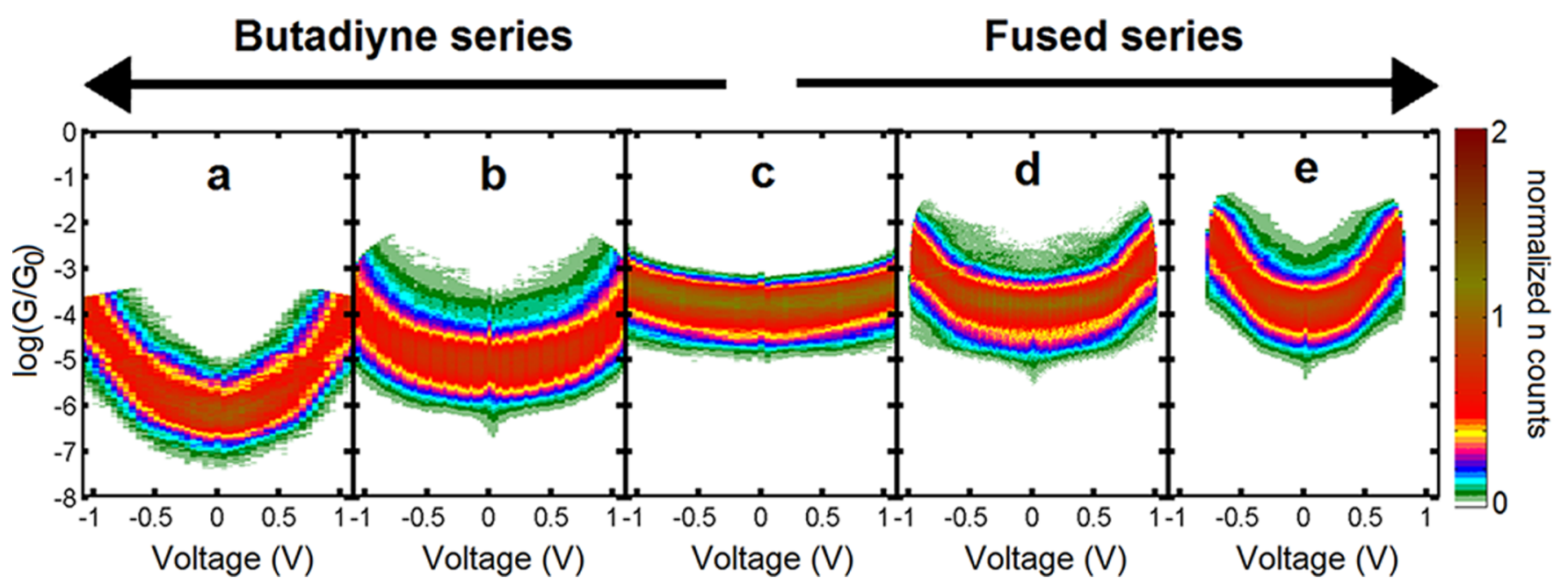

Figure 3. (a-e) $\log \left(G / G_{0}\right)-V 2 \mathrm{D}$ histograms generated from all $I-V$ traces recorded for each junction ((a) P3, (b) P2, (c) P1, (d) fP2, (e) fP3). $N_{\mathrm{IVs}}=3481$ (P3), 34855 (P2), 5233 (P1), 8737 (fP2), 13741 (fP3). $N_{\text {junctions }}=139$ (P3), 239 (P2) 177 (P1) 266 (fP2), 283 (fP3).
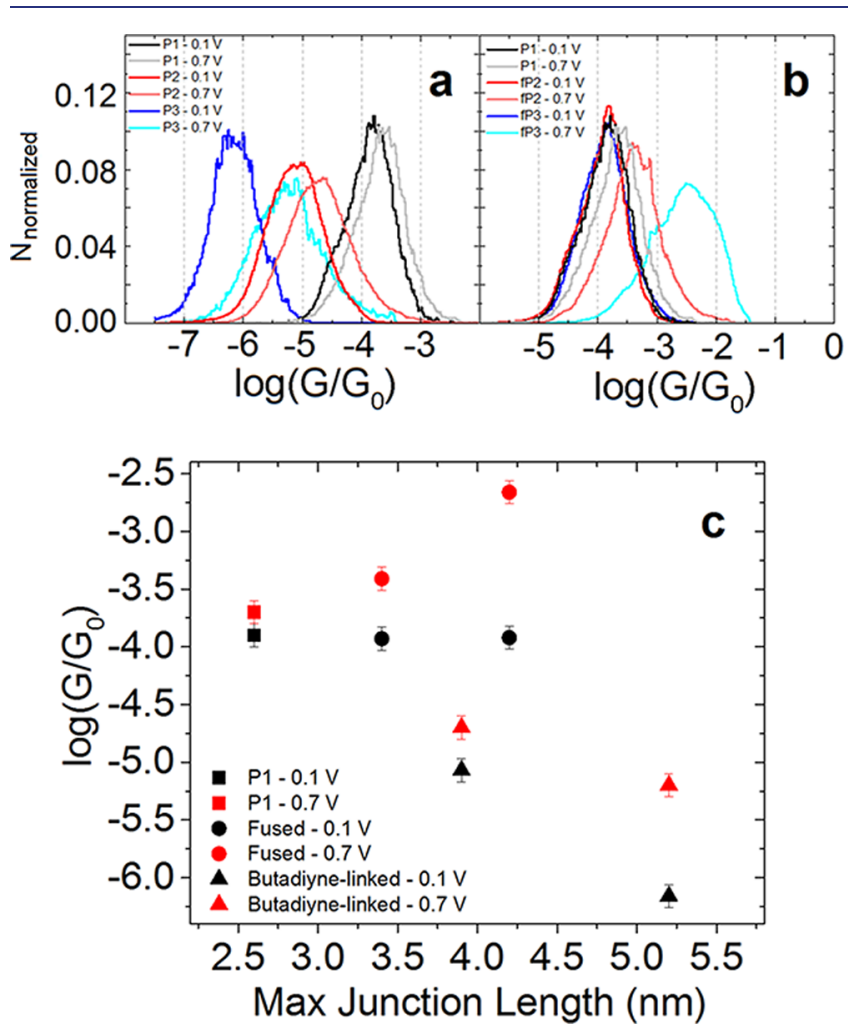

Figure 4. $(a$ and $b$ ) $1 D$ conductance histograms constructed from the data obtained at 0.1 and $0.7 \mathrm{~V}$ for P1, P2, P3 and P1, fP2, fP3 respectively. (c) Conductance peak positions at 0.1 and $0.7 \mathrm{~V}$ for each compound plotted versus the maximum expected junction length (measured between two Au atoms bonded to the terminal S atoms). For the butadiyne series, the value of $\beta$ decreases from $2.0( \pm 0.04)$ $\mathrm{nm}^{-1}$ at $V=0$ to $1.4( \pm 0.3) \mathrm{nm}^{-1}$ at $V=0.7$, reaching $0.9( \pm 0.3)$ $\mathrm{nm}^{-1}$ at $V=0.9$. For the fused series, $\beta$ is very close to $0 \mathrm{~nm}^{-1}$ at $V=$ 0 , and decreases to $-1.5( \pm 0.4) \mathrm{nm}^{-1}$ at $V=0.7$.

is discussed further in Section 2.4 of the Supporting Information). At $0.7 \mathrm{~V}$, it is clear, however, that the conductance trend now becomes $G_{\mathrm{fP} 3}>G_{\mathrm{fP} 2}>G_{\mathrm{P} 1}$ due to the dependence of $G$ on $V$ increasing in the order $\mathbf{P 1}<\mathrm{fP2}<$ fP3. Between 0.1 and $0.3 \mathrm{~V}$, there is no clear conductance trend, and this can be seen more clearly in the mean $\log (G /$
$\left.G_{0}\right)-V$ in Figure 5c. At $0.7 \mathrm{~V}$, the conductance of $\mathrm{fP} 3$ is not only 2.5 orders of magnitude larger than $\mathbf{P} 3$ but also around a factor 20 larger than P1.

In the case of fP3, and unlike for the butadiyne series, if the bias range is increased to $\pm 1.0 \mathrm{~V}$, or beyond, the general behavior of the $G-V$ traces changes significantly. Molecular junction stability seems largely unaffected, and plateaus can persist to the same lengths as previously (see Figure S25 for an example). We can also still observe ' $U$ '-shaped $\log \left(G / G_{0}\right)-V$ traces as before, some of which display clear evidence of leveling-off at high bias (examples are shown in Figure S20). On the other hand, many other junctions display $\log \left(G / G_{0}\right)-$ $V$ traces with completely different shapes, with some examples shown in Figures S21 and S22. Some junctions display traces with a 'V'-shaped profile, whereas others have pronounced flat regions, but with a dip around zero-bias. As this is clearly a voltage-related effect, and as the traces no longer fit with the shape expected in the off-resonant transport regime, we deduce that the molecule becomes temporarily charged due to the proximity of molecular levels to the Fermi level of (at least one of) the electrodes. We have measured the electrochemical HOMO-LUMO gap of fP3 to be $0.78 \mathrm{eV}$ (see Figure S2), which means that application of $1 \mathrm{~V}$ can plausibly bring the molecule into resonance. As this effect is only seen when the bias is ramped to $1 \mathrm{~V}$ and above (in this sample), then in the low-bias regime we assume transport takes place through a formally neutral molecule. In a separate experimental run on a fresh sample, we have noticed that the onset of this behavior can be slightly lower (see Figures S23 and S24), which can be explained by small fluctuations in the mean value of $E_{\mathrm{F}}$ between samples, and ties in with the findings in Figure S7. Below this "critical" voltage, however, transport can still feasibly occur via a tunneling or hopping process, as the length of fP3 $(4 \mathrm{~nm})$ coincides with the typical crossover length between these two regimes. ${ }^{15}$ As we shall show, however, the behavior of both the fused and $\mathrm{C} 4$ series can be adequately modeled in terms of a coherent (purely tunneling) transport mechanism over all bias voltages explored $( \pm 1 \mathrm{~V})$.

For the butadiyne series, despite the strong $G-V$ dependence of P3, the conductance values do not cross over the $1 \mathrm{~V}$ range studied and, as the values decay exponentially, we can plot the $\beta$ value as described in eq 1 between low and high bias 

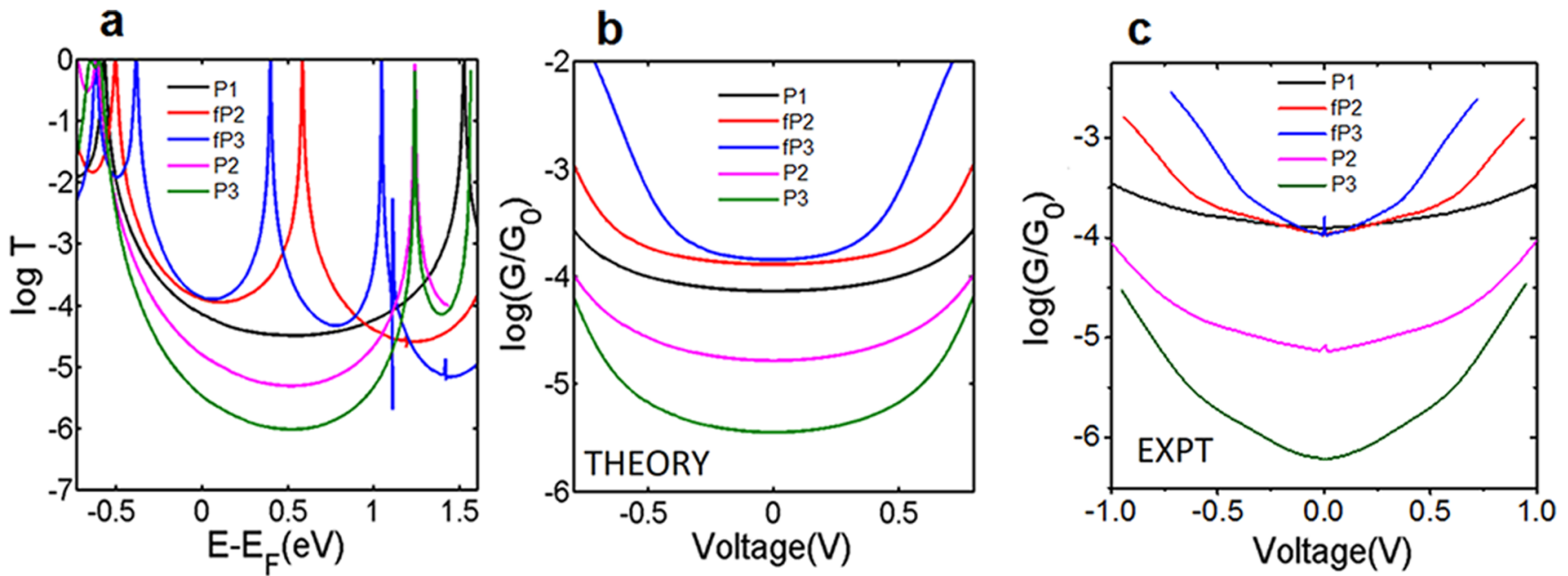

Figure 5. (a) Calculated transmission coefficient using mean field Hamiltonian obtained from DFT for fused and butadiyne-linked porphyrin series. (b) Calculated conductance vs bias voltage for each compound (c) Mean experimental $\log \left(G / G_{0}\right)$ traces for each compound.

(black squares in Figure S18b, with the $\ln G$ plots at each voltage shown in S18a). Specifically, we find values of 2.0 $( \pm 0.04) \mathrm{nm}^{-1}$ at zero bias and $0.9( \pm 0.3) \mathrm{nm}^{-1}$ at $0.9 \mathrm{~V}$. Our previous measurements on these molecules also yielded a low beta value at moderate bias $\left(0.4 \mathrm{~nm}^{-1}\right.$ at $\left.0.6 \mathrm{~V}\right)$, using a different technique, namely the STM-based $I(s)$ method. $^{40}$ Despite the small size of these values, tunnelling again appears to be the dominant mechanism in C4-linked porphyrins (as suggested in references 12,41 where $\beta$ was measured to be 0.3 and $0.4 \mathrm{~nm}^{-1}$, respectively).

DFT Transport Calculations. To elucidate the underlying transport mechanisms leading to the observed voltage dependence, we used density functional theory combined with the quantum transport code Gollum to compute the conductance versus voltage of both the fused and C4-linked molecules (see Section 4 in the Supporting Information for more details). The resulting transmission curves are shown in Figure 5a, and the corresponding conductance versus voltage curves are presented in Figures $5 \mathrm{~b}$. In the case of the fused porphyrins, the Fermi level lies in the tail of their nondegenerate HOMOs and the HOMO dominated transport is obtained. As expected the conductance increases with voltage for all molecules. At zero bias, $G_{\mathbf{P} 3} \ll G_{\mathbf{P} 2} \ll G_{\mathrm{P} 1}$, whereas for the fused series, the conductance values lie within a factor two of each other, consistent with the experiments (Figure 5c). As the bias is then increased beyond $0.5 \mathrm{~V}$, the following trend is obtained: conductance of $G_{\mathrm{fP} 3} \gg G_{\mathrm{fP} 2} \gg G_{\mathrm{P} 1}$, which is in stark contrast to the C4-linked series where the behavior remains $G_{\mathbf{P} 3}<G_{\mathbf{P 2}}<G_{\mathbf{P} 1}$. This again is in good agreement with the experimental values. Figure S32 of the Supporting Information shows the calculated energies of the HOMO, HOMO-1, LUMO, LUMO+1. In the case of the $\mathrm{C} 4$ series, in which the porphyrins are only moderately coupled, the HOMOs/ LUMOs are almost degenerate. For the more strongly coupled fused porphyrins this is not the case, and the HOMO-LUMO gap decreases rapidly with length.

To model the $\beta-V$ behavior of the butadiyne series, we have also considered a simple tight binding (TB) scheme, complementing the DFT calculations. The full details of the model are presented in Section 3 of the Supporting Information, but briefly we treat each porphyrin ring as a single hopping site, $\varepsilon$, coupled through a hopping element $\gamma$ and connected to the leads through sites $\varepsilon_{\mathrm{L}}$ and $\varepsilon_{\mathrm{R}}$. If we set $\gamma$ $=0.04 \mathrm{eV}$, which is the shift in the electrochemical (EC) oxidation potential between the monomer and $\operatorname{dimer}^{14}$ (as we assume HOMO dominated transport due to the thiol anchors ${ }^{42}$ ), and $\varepsilon=-0.8 \mathrm{eV}$, then we find a low bias $\beta$ value of $4 \mathrm{~nm}^{-1}$, about a factor 1.5-2 larger than measured. Increasing $\gamma$ to $0.17 \mathrm{eV}$ provides a better fit. We have also calculated the conductance as a function of voltage, $G(V)$, in order to plot $\beta-V$. Figure S30 shows these results, where the data from the TB model (using $\gamma=0.17 \mathrm{eV}$ ) closely follow the experimental data. At a basic level, this model helps to explain the differences between C4-linked and fused porphyrins, as the strong increase in intersite coupling arising through ring fusion is clearly expected to reduce $\beta$. A complete description of the fused series, however, requires both HOMO and LUMO states to be considered, which is appropriately described by our DFT calculations. The key point, however, is to demonstrate that the variation of $\beta$ with voltage will not be unique to porphyrins, but rather should be intrinsic to any chain of repeating units (phenyl, thiophene etc.). This understanding will facilitate the rational design of different molecular wires with low, or even negative- $\beta$, which is important for achieving long-range charge transport.

The strong change in HOMO/LUMO energy along the fused series causes the effective barrier for electrons to change significantly as the length increases. This logically implies that there is no single degree of attenuation per unit length for the fused porphyrins. This is, technically speaking, also the case for the $\mathrm{C} 4$ series, although as the change in effective barrier is much less as a function of length here, it can essentially be ignored. This consideration highlights that $\beta$ (as commonly defined $^{21}$ ) is an approximation when applied to molecular oligomers, useful only when the effective barrier remains practically constant with length.

\section{CONCLUSIONS}

Overall, our results demonstrate that fused porphyrin tapes substantially increase in conductance with length at moderate bias voltages, by more than a factor 10 . This is the first time that this behavior has been observed in molecular wires, as well as more generally for atomic-scale junctions. This phenomenon is caused by a large decrease in the HOMO-LUMO gap 
with length, which compensates for the increased tunneling distance. In contrast, for the series of moderately coupled butadiyne-linked wires, the conductance decays exponentially over a wide range of bias voltages, with the degree of attenuation reducing as the voltage increase. Both series strongly indicate coherent transport as the dominant mechanism. The counterintuitive conductance increase with length observed in the fused porphyrins should be a generic effect, and it is likely to occur in other strongly coupled systems, such as acenes.

\section{ASSOCIATED CONTENT}

\section{S Supporting Information}

The Supporting Information is available free of charge on the ACS Publications website at DOI: 10.1021/jacs.8b06338.

All synthetic procedures and analysis, general methods for the BJ study plus further analysis, tight binding theory, DFT calculations of orbital energies and further transmission calculations (PDF)

\section{AUTHOR INFORMATION}

\section{Corresponding Authors}

*E.Leary@liverpool.ac.uk

*harry.anderson@chem.ox.ac.uk

*c.lambert@lancaster.ac.uk

*nichols@liverpool.ac.uk

\section{ORCID $\odot$}

Edmund Leary: 0000-0001-7541-5997

Gabino Rubio-Bollinger: 0000-0001-7864-8980

Hatef Sadeghi: 0000-0001-5398-8620

Andrew Hodgson: 0000-0001-8677-7467

Colin J. Lambert: 0000-0003-2332-9610

Harry L. Anderson: 0000-0002-1801-8132

Notes

The authors declare no competing financial interest.

All individual traces $(G-z$ and $I-V)$ for the STM experiments are freely available after an embargo date of 04/25/19 at the following repository address: 10.17638/datacat.liverpool.ac.uk/462.

\section{ACKNOWLEDGMENTS}

R.J.N., S.H., E.L. and A.H. acknowledge the UK Engineering and Physical Sciences Research Council (EPSRC) for the funding of this research under grant numbers EP/M014169/1, P/M029522/1 and EP/M005046/1. N.A., C.L., S.S. and I.G. acknowledge the European Commission (EC) FP7 ITN "MOLESCO" (project no. 606728) and the EPSRC (grant nos. EP/M014452/1 and EP/N017188/1). H.L.A. thanks the EPSRC (grants EP/N017188/1, EP/D076552/1 and EP/ M016110/1), UK National Mass Spectrometry Facility at Swansea University, for recording mass spectra. H.S. acknowledges the Leverhulme Trust for Leverhulme Early Career Fellowship no. ECF-2017-186. S.S. acknowledges the Leverhulme Trust for Leverhulme Early Career Fellowship no. ECF-2018-375. N.A. and G.R.B. acknowledge the Spanish MINECO (Grant MAT2014-57915-R, MAT2017-88693-R and MDM-2014-0377) and Comunidad de Madrid (Grant NANOFRONTMAG-CM, S2013/MIT-2850). N.A. and C.L. have received funding from the European Union's Horizon 2020 research and innovation programme under grant agreement no. 767187. IMDEA Nanociencia acknowledges support from the 'Severo Ochoa' Programme for Centres of Excellence in R\&D (MINECO, Grant SEV-2016-0686).

\section{REFERENCES}

(1) Scholes, G. D.; Fleming, G. R.; Olaya-Castro, A.; Van Grondelle, R. Nat. Chem. 2011, 3, 763.

(2) Amdursky, N.; Marchak, D.; Sepunaru, L.; Pecht, I.; Sheves, M.; Cahen, D. Adv. Mater. 2014, 26, 7142-7161.

(3) Ruiz, M. P.; Aragonès, A. C.; Camarero, N.; Vilhena, J.; Ortega, M.; Zotti, L. A.; Pérez, R.; Cuevas, J. C.; Gorostiza, P.; Díez-Pérez, I. J. Am. Chem. Soc. 2017, 139, 15337-15346.

(4) Li, Z.; Li, H.; Chen, S.; Froehlich, T.; Yi, C.; Schönenberger, C.; Calame, M.; Decurtins, S.; Liu, S.-X.; Borguet, E. J. Am. Chem. Soc. 2014, 136, 8867-8870.

(5) Kim, Y.; Hellmuth, T. J.; Sysoiev, D.; Pauly, F.; Pietsch, T.; Wolf, J.; Erbe, A.; Huhn, T.; Groth, U.; Steiner, U. E.; Scheer, E.; et al. Nano Lett. 2012, 12, 3736-3742.

(6) Frisbie, C. D. Science 2016, 352, 1394-1395.

(7) Darwish, N.; Díez-Pérez, I.; Guo, S.; Tao, N.; Gooding, J. J.; Paddon-Row, M. N. J. Phys. Chem. C 2012, 116, 21093-21097.

(8) Zhou, J.; Wang, K.; Xu, B.; Dubi, Y. J. Am. Chem. Soc. 2018, 140, $70-73$.

(9) Evangeli, C.; Gillemot, K.; Leary, E.; González, M. T.; RubioBollinger, G.; Lambert, C. J.; Agraï, N. Nano Lett. 2013, 13, 21412145.

(10) Rincón-García, L.; Evangeli, C.; Rubio-Bollinger, G.; Agraït, N. Chem. Soc. Rev. 2016, 45, 4285-4306.

(11) Cui, L.; Miao, R.; Wang, K.; Thompson, D.; Zotti, L. A.; Cuevas, J. C.; Meyhofer, E.; Reddy, P. Nat. Nanotechnol. 2018, 13, $122-127$.

(12) Sedghi, G.; García-Suárez, V. M.; Esdaile, L. J.; Anderson, H. L.; Lambert, C. J.; Martín, S.; Bethell, D.; Higgins, S. J.; Elliott, M.; Bennett, N.; Macdonald, E.; Nichols, R. J. Nat. Nanotechnol. 2011, 6, 517.

(13) Sedghi, G.; Esdaile, L. J.; Anderson, H. L.; Martin, S.; Bethell, D.; Higgins, S. J.; Nichols, R. J. Adv. Mater. 2012, 24, 653-657.

(14) Winters, M. U.; Dahlstedt, E.; Blades, H. E.; Wilson, C. J.; Frampton, M. J.; Anderson, H. L.; Albinsson, B. J. Am. Chem. Soc. 2007, 129, 4291-4297.

(15) Luo, L.; Choi, S. H.; Frisbie, C. D. Chem. Mater. 2011, 23, 631-645.

(16) Kuang, G.; Chen, S. Z.; Yan, L.; Chen, K. Q.; Shang, X.; Liu, P. N.; Lin, N. J. Am. Chem. Soc. 2018, 140, 570-573.

(17) Li, Z.; Park, T.-H.; Rawson, J.; Therien, M. J.; Borguet, E. Nano Lett. 2012, 12, 2722-2727.

(18) Tanaka, T.; Osuka, A. Chem. Soc. Rev. 2015, 44, 943-969.

(19) Li, Z.; Smeu, M.; Ratner, M. A.; Borguet, E. J. Phys. Chem. C 2013, 117, 14890-14898.

(20) Kiguchi, M.; Takahashi, T.; Kanehara, M.; Teranishi, T.; Murakoshi, K. J. Phys. Chem. C 2009, 113, 9014-9017.

(21) Perrin, M. L.; Verzijl, C. J. O.; Martin, C. A.; Shaikh, A. J.; Eelkema, R.; van Esch, J. H.; van Ruitenbeek, J. M.; Thijssen, J. M.; van Der Zant, H. S. J.; Dulić, D. Nat. Nanotechnol. 2013, 8, 282.

(22) Holten, D.; Bocian, D. F.; Lindsey, J. S. Acc. Chem. Res. 2002, $35,57-69$.

(23) Taylor, P. N.; Huuskonen, J.; Aplin, R. T.; Anderson, H. L.; Rumbles, G.; Williams, E.; Huuskonen, J. Chem. Commun. 1998, No. 8, 909-910.

(24) Anderson, H. L. Inorg. Chem. 1994, 33, 972-981.

(25) Lin, V. S.; DiMagno, S. G.; Therien, M. J. Science 1994, 264, 1105-1111.

(26) Tsuda, A.; Osuka, A. Science 2001, 293, 79-82.

(27) Kang, B. K.; Aratani, N.; Lim, J. K.; Kim, D.; Osuka, A.; Yoo, K.-H. Chem. Phys. Lett. 2005, 412, 303-306.

(28) Algethami, N.; Sadeghi, H.; Sangtarash, S.; Lambert, C. J. Nano Lett. 2018, 18, 4482-4486.

(29) Wang, W.; Lee, T.; Reed, M. A. Phys. Rev. B: Condens. Matter Mater. Phys. 2003, 68, No. 035416. 
(30) Koch, M.; Ample, F.; Joachim, C.; Grill, L. Nat. Nanotechnol. 2012, 7, 713.

(31) Kuang, G.; Chen, S.-Z.; Wang, W.; Lin, T.; Chen, K.; Shang, X.; Liu, P. N.; Lin, N. J. Am. Chem. Soc. 2016, 138, 11140-11143.

(32) Arroyo, C. R.; Leary, E.; Castellanos-Gomez, A.; RubioBollinger, G.; Gonzalez, M. T.; Agrait, N. J. Am. Chem. Soc. 2011, 133, 14313-14319.

(33) Li, C.; Pobelov, I.; Wandlowski, T.; Bagrets, A.; Arnold, A.; Evers, F. J. Am. Chem. Soc. 2008, 130, 318-326.

(34) Venkataraman, L.; Klare, J. E.; Nuckolls, C.; Hybertsen, M. S.; Steigerwald, M. L. Nature 2006, 442, 904.

(35) Kaliginedi, V.; Moreno-Garcia, P.; Valkenier, H.; Hong, W.; Garcia-Suarez, V. M.; Buiter, P.; Otten, J. L. H.; Hummelen, J. C.; Lambert, C. J.; Wandlowski, T. J. Am. Chem. Soc. 2012, 134, 52625275.

(36) Valkenier, H.; Huisman, E. H.; van Hal, P. A.; de Leeuw, D. M.; Chiechi, R. C.; Hummelen, J. C. J. Am. Chem. Soc. 2011, 133, 49304939.

(37) Li, Z.; Smeu, M.; Park, T.-H.; Rawson, J.; Xing, Y.; Therien, M. J.; Ratner, M. A.; Borguet, E. Nano Lett. 2014, 14, 5493-5499.

(38) Leary, E.; Roche, C.; Jiang, H.-W.; Grace, I.; González, M. T.; Rubio-Bollinger, G.; Romero-Muñiz, C.; Xiong, Y.; Al-Galiby, Q.; Noori, M.; Lebedeva, M. A.; Porfyrakis, K.; Agrait, N.; Hodgson, A.; Higgins, S. J.; Lambert, C. J.; Anderson, H. L.; Nichols, R. J. J. Am. Chem. Soc. 2018, 140, 710-718.

(39) Gonzalez, M. T.; Leary, E.; García, R.; Verma, P.; Herranz, M. A.; Rubio-Bollinger, G.; Martín, N.; Agraït, N. J. Phys. Chem. C 2011, 115, 17973-17978.

(40) Sedghi, G.; Sawada, K.; Esdaile, L. J.; Hoffmann, M.; Anderson, H. L.; Bethell, D.; Haiss, W.; Higgins, S. J.; Nichols, R. J. J. Am. Chem. Soc. 2008, 130, 8582-8583.

(41) Gilbert Gatty, M.; Kahnt, A.; Esdaile, L. J.; Hutin, M.; Anderson, H. L.; Albinsson, B. J. Phys. Chem. B 2015, 119, 75987611.

(42) Reddy, P.; Jang, S.-Y.; Segalman, R. A.; Majumdar, A. Science 2007, 315, 1568-1571. 